\title{
Effects of Constraint-Induced Therapy Versus Bilateral Arm Training on Motor Performance, Daily Functions, and Quality of Life in Stroke Survivors
}

\author{
Keh-chung Lin, ScD, OTR, Ya-fen Chang, MS, Ching-yi Wu, ScD, OTR, and Yi-an Chen, MS
}

\begin{abstract}
Background and Objective. This study investigated the relative effects of distributed constraint-induced therapy (CIT) and bilateral arm training (BAT) on motor performance, daily function, functional use of the affected arm, and quality of life in patients with hemiparetic stroke. Methods. A total of 60 patients were randomized to distributed CIT, BAT, or a control intervention of less specific but active therapy. Each group received intensive training for 2 hours/day, 5 days/week, for 3 weeks. Pretreatment and posttreatment measures included the Fugl-Meyer Assessment (FMA), Functional Independence Measure (FIM), Motor Activity Log (MAL), and Stroke Impact Scale (SIS). The proximal and distal scores of FMA were used to examine separate upper limb (UL) elements of movement. Results. The distributed CIT and BAT groups showed better performance in the overall and the distal part score of the FMA than the control group. The BAT group exhibited greater gains in the proximal part score of the FMA than the distributed CIT and control groups. Enhanced performance was found for the distributed CIT group in the MAL, the subtest of locomotion in the FIM, and certain domains of the SIS (eg, ADL/IADL). Conclusion. BAT may uniquely improve proximal UL motor impairment. In contrast, distributed CIT may produce greater functional gains for the affected UL in subjects with mild to moderate chronic hemiparesis.
\end{abstract}

Keywords: Controlled clinical trial; Stroke rehabilitation; Constraint-induced therapy; Bilateral arm training; Upper extremity; Quality of life

U pper limb (UL) hemiparesis is a major factor restricting functional recovery in more than $85 \%$ of stroke patients. ${ }^{1,2}$ Unsuccessful use of the affected UL in stroke patients may cause "learned nonuse phenomenon," in which patients habitually rely on their unaffected UL to accomplish daily activities. ${ }^{3}$ Constraintinduced therapy $(\mathrm{CIT})^{4,5}$ has been found to be effective for overcoming learned nonuse. ${ }^{2}$ CIT involves restraint of the unaffected UL over an extended period of time (90\% of waking hours) in combination with repetition of task-specific intensive training of the affected UL ( 6 hours/session, 5 sessions/week, for 2 weeks)., ${ }^{3,5}$ However, such an intensive training schedule might not be acceptable for many patients. ${ }^{6}$ Distributed or modified forms of CIT were developed..$^{7-9}$ These forms decreased the training hours for each session ( 0.5 to 3 hours/session) and restraint hours per day ( 5 to 9 hours/day), and distributed these sessions to a long duration ( 3 to 10 weeks). Several studies have demonstrated the benefits of CIT or its derivatives (distributed or modified CIT), relative to traditional rehabilitation or control intervention, in improving motor capacity, functional performance, and quality of life..$^{3-5,8-12}$
An alternative treatment program that is gaining increasing attention is bilateral arm training (BAT), which employs the repetitive practice of symmetrical bilateral movement ( 0.3 to 2.25 hours/day, 3 to 5 days/week, for 2 to 8 weeks) to improve motor performance of the affected UL. ${ }^{13,14}$ There are a variety of forms of BAT. ${ }^{15}$ Some involve robot-assisted and repetitive movement training, ${ }^{13,16-18}$ whereas others employ repetitive practice of functional tasks. ${ }^{19-22}$ Some studies have revealed that BAT reduces UL impairment (evaluated by Fugl-Meyer Assessment [FMA]) when compared with traditional rehabilitation, ${ }^{13,17,21-23}$ whereas others claim that BAT did not confer benefits on performance of impaired $\mathrm{UL}^{18,21,22}$ and had limited effects on functional independence ${ }^{19,23}$ and spontaneous use of the affected hand. ${ }^{18}$ Small sample sizes, differential degrees of initial impairment, and insufficient intensity of treatment may each have contributed to the lack of significant effects.

Distributed CIT and BAT share similar key therapeutic elements (mass and repetitive practice with specific techniques), and both target improvement of the affected UL, although at first glance, the concept of BAT seems contradictory to the

From the School of Occupational Therapy, College of Medicine, National Taiwan University, and Division of Occupational Therapy, Department of Physical Medicine and Rehabilitation, National Taiwan University Hospital, Taipei (K-CL); Division of Occupational Therapy, Department of Rehabilitation, Shin Kong Wu Ho-Su Memorial Hospital, Taipei (Y-FC); Graduate Institute of Clinical Behavioral Science and Department of Occupational Therapy, Chang Gung University, Taoyuan (C-YW); and Department of Occupational Therapy, Yung Cheng Rehabilitation Clinic, Taipei (Y-AC), Taiwan. Address correspondence to Ching-yi Wu, ScD, OTR, Graduate Institute of Clinical Behavioral Science and Department of Occupational Therapy, Chang Gung University, 259 Wen-hwa 1st Road, Kwei-shan, Taoyuan, Taiwan. E-mail: cywu@mail.cgu.edu.tw. 
concept of distributed CIT. ${ }^{24,25}$ It remains unclear whether BAT could be an alternative program through which to overcome the phenomenon of learned nonuse. ${ }^{18}$ In addition, Whitall ${ }^{25}$ claimed that exploring the relative effects of different researchsupported training programs is important to the design and development of efficient and effective rehabilitation programs for stroke patients. To achieve this aim, there is a need for rigorous comparison between distributed CIT and BAT. ${ }^{26,27}$

Although some investigations have examined the differences in treatment outcomes between unilateral and bilateral trainings, ${ }^{14,22,28}$ no research to date has studied the relative effects of distributed CIT versus BAT regarding to what extent and in what situations one approach may be more beneficial than the other. This study compared the relative effects of distributed CIT versus BAT versus control intervention on motor capacity, functional performance, and quality of life. We hypothesized that both distributed CIT and BAT would elicit better performance than control intervention. In addition, distributed CIT and BAT may produce differential benefits regarding specific outcome measures (eg, motor function, use of the affected arm in real-world situation, etc).

\section{Method}

\section{Participants}

A total of 60 patients ( 34 men and 26 women) with a mean age of 52.14 years (range, 23 to 81 years), with unilateral stroke identified by brain imaging, were recruited for the study. All patients attended the outpatient rehabilitation programs when admitted to the study and signed informed consent forms approved by the Institutional Review Board. Participants received independent examinations by an occupational therapist to determine their eligibility for inclusion according to the following criteria: $(a)$ more than 6 months post onset of cerebrovascular accident of ischemic or hemorrhagic type; (b) Brunnstrom stage above III for proximal and distal parts of UL ${ }^{29} ;(c)$ considerable nonuse of the affected UL (an amount of use score $<2.5$ on the Motor Activity Log $[\mathrm{MAL}])^{30} ;(d)$ no serious cognitive deficits (a score $\geq 24$ on the Mini-Mental State Examination [MMSE] $)^{31} ;(e)$ no excessive spasticity in any joints of the affected UL (shoulder, elbow, wrist, or fingers; Modified Ashworth Scale [MAS] score $\leq 2$ in any joint); $(f)$ lack of participation in any experimental rehabilitation or drug studies within the past 6 months; and $(g)$ no balance problems sufficient to compromise safety when wearing the constraint mitt. Participants were blind to the study hypotheses.

\section{Design}

This study was a randomized pretest and posttest control group design. Sixty participants were individually randomized into the distributed CIT, BAT, or control intervention groups, with the computerized (block) randomization scheme, including prestratification according to participating hospital.
One set of opaque, numbered envelopes was prepared for each site containing cards indicating the allocated group (Figure 1). When a new patient was registered, a card was extracted and the relevant occupational therapist informed of the group allocation. Consequently, each group had 20 participants. All groups received intensive training for 2 hours/ day, 5 days/week, for 3 weeks. Trainings were administered during regularly scheduled occupational therapy sessions, and all other routine interdisciplinary stroke rehabilitation was continued as usual. Five therapists were trained by the primary investigators to provide consistent therapy. The training included group review of pertinent CIT and BAT literature and validation of therapists' videotaped responses to various clinical presentations.

Before and after the 3-week intervention period, clinical evaluations (FMA, Functional Independence Measure [FIM], MAL, and Stroke Impact Scale [SIS]) were administered by 3 certified occupational therapists. These 3 raters were blinded to the participant group and trained to properly administer the outcome measures. This training included careful examination of written instructions and repeated practice. Rater competence was assessed by a senior certified occupational therapist. After the 3 raters finished the assessment for all participants, we calculated the post hoc interrater reliability for FMA and FIM, and obtained high reliability (intraclass correlation coefficients $^{32}>.95$ for both measures).

\section{Interventions}

The distributed CIT group focused on restriction of movement of the unaffected hand by placement of the hand in a mitt for 6 hours/day and intensive training of the affected UL in functional tasks for 2 hours/weekday, including reaching forward or upward to move a cup, picking up coins, picking up a utensil to take food, grasping and releasing various blocks, and other functional movements involved in daily activities. The level of challenge was adapted based on patient ability and improvement during training. Hours of mitten wearing per day were recorded by the patients and confirmed by the caregivers.

The BAT group concentrated on the simultaneous movements of both the affected and unaffected UL in functional tasks in symmetric or alternating patterns for 2 hours/weekday for 3 weeks. These functional tasks also emphasized UL movements involved in daily activities, but focused on both ULs moving synchronously, such as lifting 2 cups, picking up 2 pegs, reaching forward or upward to move blocks, grasping and releasing 2 towels, and so on. This group did not perform at-home practice as the CIT group did.

The control intervention group was designed to control for the duration and intensity of patient-therapist interactions and therapeutic activities (2 hours/day, 5 days/week, for 3 weeks). Therapy in the control intervention group involved training for hand function, coordination, balance, and movements of the affected UL, as well as compensatory practice on functional tasks with the unaffected UL or both ULs. 
Figure 1

Flow Diagram of the Randomization Procedure

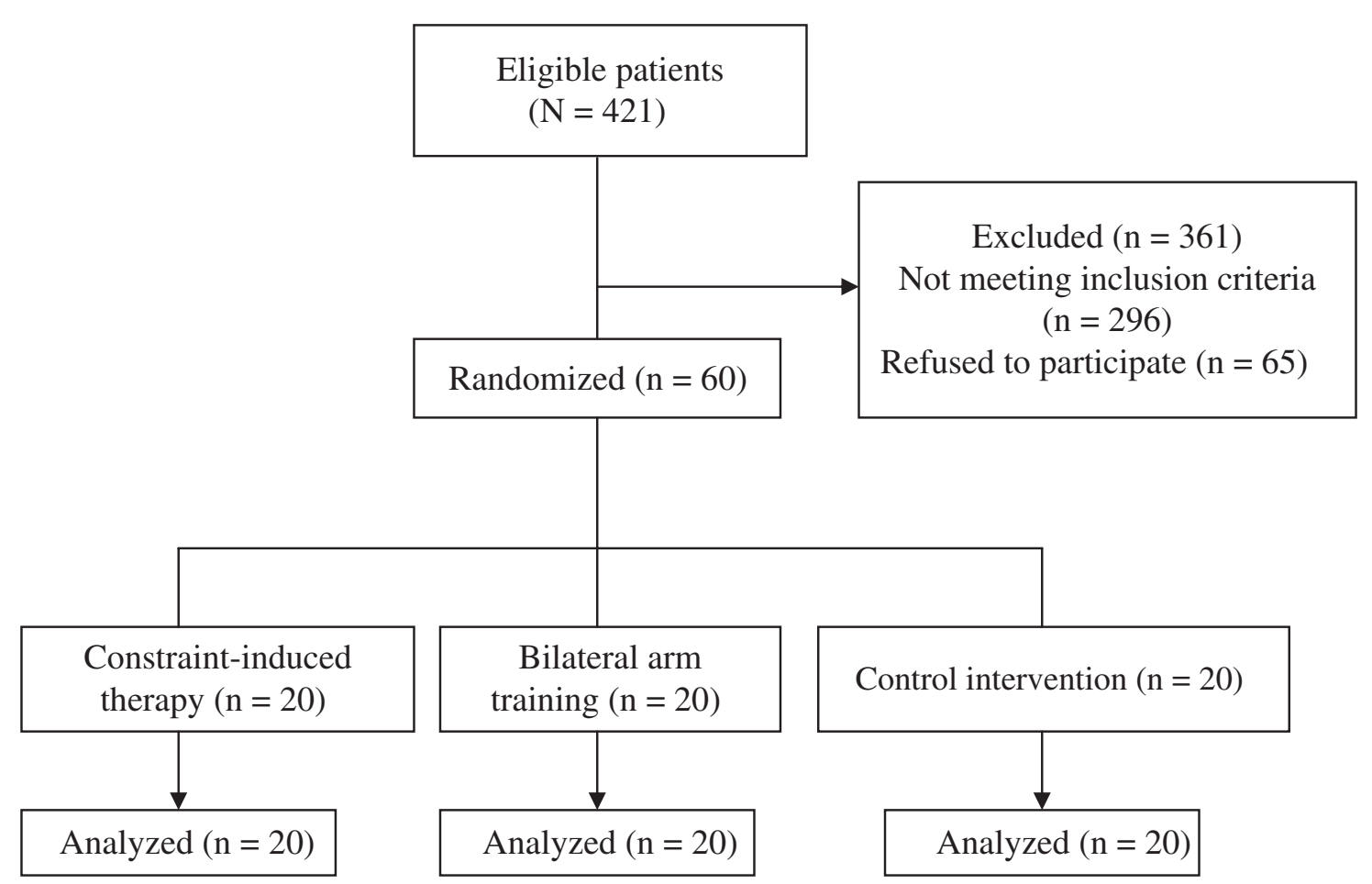

\section{Outcome Measures}

We used FMA, FIM, MAL, and SIS to evaluate motor impairment, daily function, use of the affected arm in realworld situations, and quality of life, respectively. The FMA of UL part score (maximum score, 66) assesses several dimensions of motor impairment by using a 3-point ordinal scale. Higher scores indicate better motor control. Test-retest reliability, interrater reliability, and construct validity of the test are well established. ${ }^{33,34}$ We also divided FMA into proximal (shoulder, elbow, and forearm) and distal (wrist and hand) parts to investigate the treatment effects on separate UL elements.

The FIM consists of 18 items divided into 6 subscales measuring self-care, sphincter control, transfer, locomotion, communication, and social cognition ability. Each item is rated from 1 to 7 based on the required level of assistance to perform the basic ADL (maximum score, 126). Higher scores demonstrate greater independent participation in daily activities. The FIM has good interrater reliability and validity. ${ }^{35,36}$

The MAL is a semistructured interview of patients to assess the amount of use (AOU) and quality of movement (QOM) of the affected UL in 30 important daily activities using a 6-point ordinal scale. Higher scores indicate better performance. This scale has been found to have good internal consistency, interrater reliability, and construct validity. ${ }^{37,38}$ The SIS was used to measure changes in quality of life. The SIS, version 3, is a 59-item self-report scale designed to assess 8 functional domains including strength, memory, emotion, communication, activities of daily living (ADLs)/instrumental ADLs, mobility, hand function, and participation using a 5-point ordinal scale, with established reliability and validity. ${ }^{39}$ Higher scores indicate better recovery by self-perception.

\section{Statistical Analysis}

To test the relative effects of the treatment groups, we used the analysis of covariance (ANCOVA) for each variable. The pretest score (FMA, FIM, MAL, and SIS) was treated as the covariate for controlling pretreatment differences, group (distributed CIT, BAT, and control intervention) as the independent variable, and the posttest score as the dependent variable. Because the hypotheses were all prespecified, no adjustments were made to the reported $P$ value. The effect size $r$ was calculated for each variable to index the magnitude of group differences in performance; a large effect is represented by an $r$ of at least .50, a moderate effect by an $r$ of .30, and a small effect by an $r$ of $.10{ }^{40}$ Fisher's least significant difference tests were used for post hoc comparisons between groups. Level of statistical significance was set at .05 .

\section{Results}

There were no significant differences in the demographic and clinical characteristics of participants in the 3 groups (Table 1). Table 2 shows the descriptive statistics and inferential 
Table 1

Characteristics of Study Participants

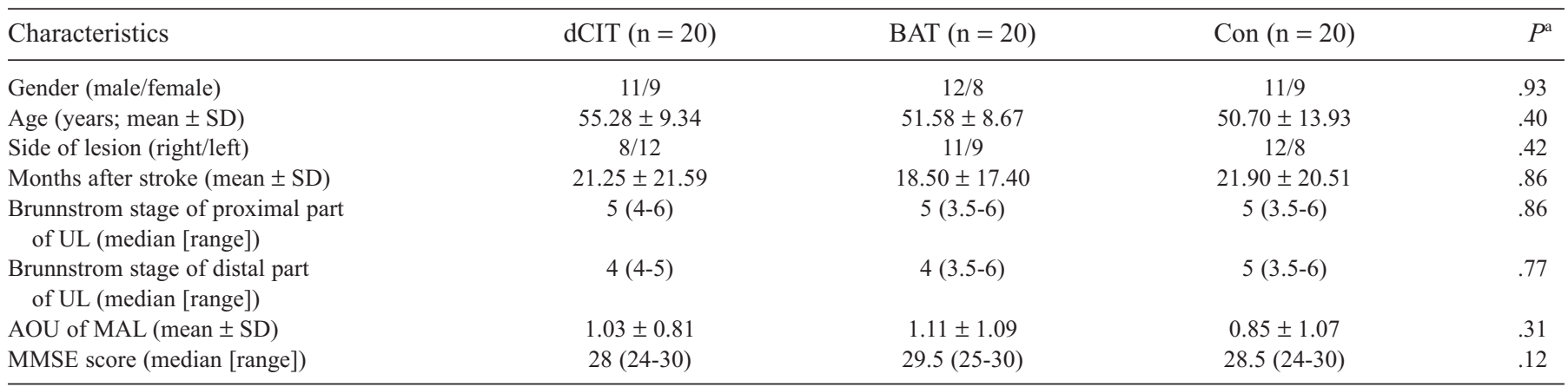

Abbreviations: dCIT, distributed constraint-induced therapy; BAT, bilateral arm training; Con, control intervention; SD, standard deviation; UL, upper limb; AOU, amount of use; MAL, Motor Activity Log; MMSE, Mini-Mental State Examination.

${ }^{a} P$ associated with $\chi^{2}$ test for categorical variables, one-way analysis of variance for continuous variables, and nonparametric test for ordinal variables.

statistics for each outcome measure. The results were partially consistent with the study hypotheses. There were differences between the 3 groups in FMA (overall score: $F(2,56)=9.72$, $P<.001, r=.51$; proximal part: $F(2,56)=3.88, P=.027$, $r=.35$; distal part: $F(2,56)=6.30, P=.003, r=.43)$ and MAL (AOU: $F(2,56)=4.77, P=.012 . r=.38$; QOM: $F(2,56)=$ $5.81, P=.005, r=.41)$. Although there was no group effect on the overall FIM score, performance on the locomotion subtest in the FIM was different between the 3 groups $(F(2,56)=$ $3.37, P=.042, r=.33)$. Differences were also found in the SIS measure (overall score: $F(2,56)=5.86, P=.005, r=.42$; ADLs/IADLs: $F(2,56)=4.94, P=.011, r=.39$; hand function: $F(2,56)=3.40, P=.040, r=.33$; participation: $F(2,56)=$ $3.92, P=.025, r=.35)$.

Post hoc analyses revealed that, in comparison with the control intervention group, distributed CIT and BAT groups each produced greater improvements in the overall FMA score $(P=.001$ for CIT vs control; $P<.001$ for BAT vs control $)$ and the distal part score of the FMA $(P=.002$ for CIT vs control; $P=.008$ for BAT vs control). The BAT group demonstrated greater improvement in the proximal part score of the FMA than the control intervention group $(P=.008)$. The distributed CIT group engendered better performance in the locomotion subtest of the FIM and both aspects of MAL than the BAT group (locomotion subtest of FIM: $P=.011$; AOU: $P=$ .042 ; QOM: $P=.004)$ and the control intervention group (locomotion subtest of FIM: $P=.037$; AOU: $P<.001$; QOM: $P=.003)$.

Post hoc analyses revealed that the distributed CIT group rated higher quality of life in the overall, ADL/IADL, and hand function domains of SIS than the control intervention group (SIS: $P=.007$; ADL/IADL domain: $P=.004$; hand function domain: $P=.015$ ). In addition, the distributed CIT group perceived better quality of life in the overall SIS and domains of ADL/IADL and social participation than the BAT group (SIS: $P=.003$; ADL/IADL domain: $P=.024$; social participation domain: $P=.009$ ).

\section{Discussion}

The findings on motor performance are in a large part consistent with our hypothesis. Both distributed CIT and BAT improved overall UL performance (overall FMA score) and distal UL (distal FMA score) to a greater extent than control intervention, which was based on neurodevelopmental therapy and compensatory practice on functional activities with the unaffected UL or both ULs. BAT demonstrated unique benefits in reducing proximal UL deficits. Partially consistent with our hypotheses, distributed CIT patients perceived improved functional use of the affected UL, as measured by AOU and QOM, and rated better scores of overall quality of life (evaluated by the SIS) and the ADL domain of the SIS than BAT and control patients. Although there was no significant difference in FIM score between the 3 groups, the distributed CIT group showed significantly better performance on the locomotion subtest after intervention than the BAT and control groups.

In agreement with previous studies, $3,4,9,12,13,17,23$ distributed CIT and BAT demonstrated greater improvement in the performance of overall UL and distal UL than control intervention. Both distributed CIT and BAT emphasize mass practice on functional tasks that may provide sufficient proprioceptive and visual feedback to reacquire motor skills. ${ }^{41}$ The beneficial effects of distributed CIT or BAT on FMA scores did not corroborate some previous studies. ${ }^{8,18,21,22}$ The primary reason may be the small sample sizes used in the previous studies. Differences in treatment intensity ${ }^{8,21}$ and treatment forms ${ }^{18,22}$ may be additional factors. Page et $\mathrm{al}^{8}$ administered modified CIT in 30-minute therapy sessions occurring 3 days per week for 10 weeks and Mudie and Matyas ${ }^{21}$ provided only 30 minutes for BAT training. Their treatment intensity was lower than that used in the present study. Richards et $\mathrm{a}^{18}$ employed robot-assisted BAT and Lewis and Byblow ${ }^{22}$ employed only 3 treatment activities for bilateral practice. Their treatment protocols were different from ours that involved nonrobotic bilateral training with a variety of functional activities. 


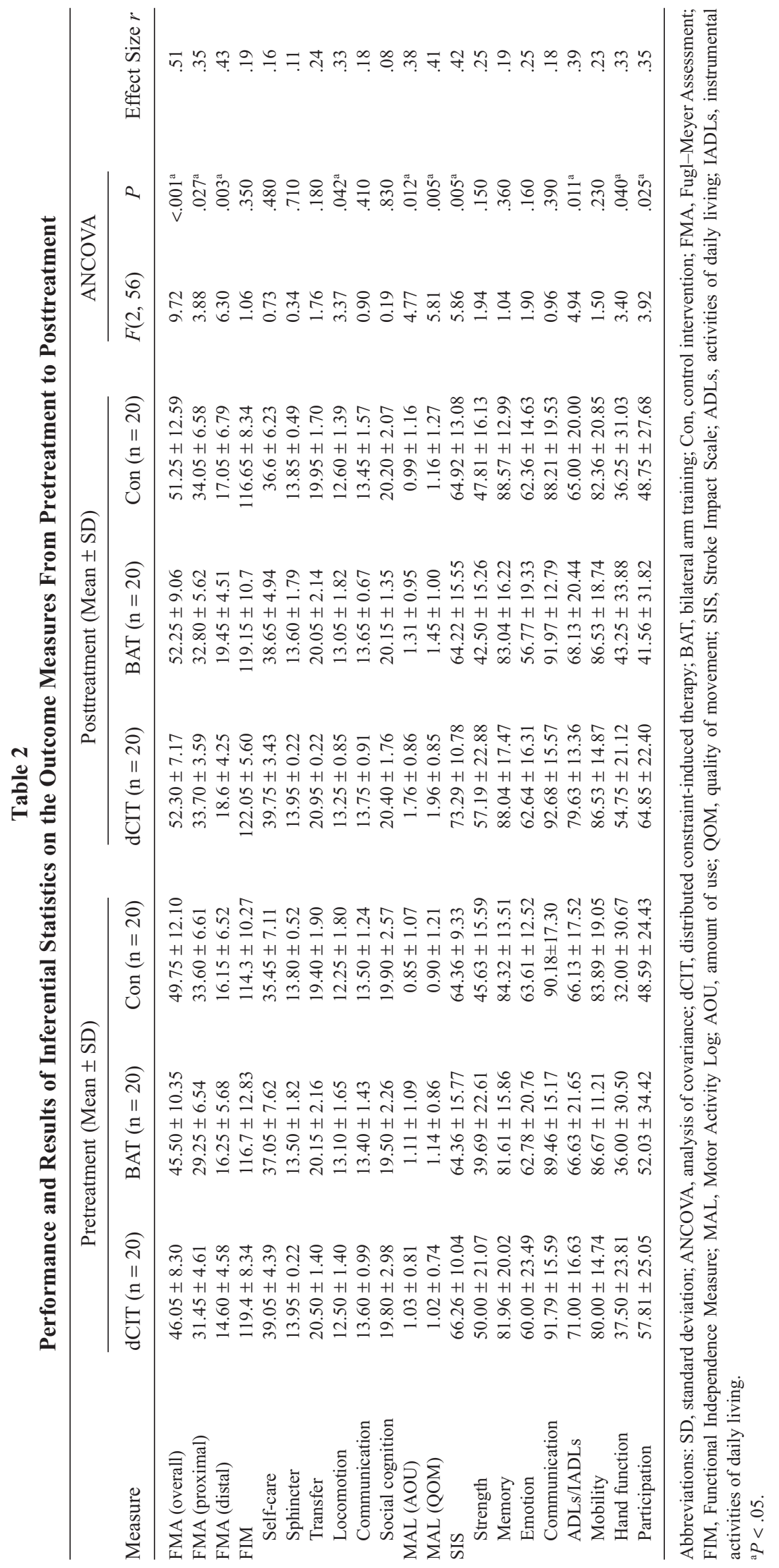


Only BAT produced significantly better motor improvements in the proximal UL than control intervention. Simultaneous activation of both hands may reduce intracortical inhibition and increase intracortical facilitation in both hemispheres and may therefore cause an additional facilitation in the affected hemisphere compared to activation of the affected hand alone. ${ }^{42,43}$ McCombe Waller and Whitall ${ }^{26}$ proposed that this speculated neural effect for the affected hemisphere may have positive aftereffects for unilateral paretic movement and improve motor skills. In summary, although both distributed CIT and BAT demonstrated greater improvements in motor capacity of UL than control intervention, BAT appears to be superior to distributed CIT in improving motor impairments of the proximal UL.

As found in previous studies, ${ }^{4,5,8,9,11,12}$ the distributed CIT led to greater improvements in the scores of the MAL (both AOU and QOM), the SIS, or the subdomains of the SIS related to paretic upper extremity function than control intervention. Restraint of the unaffected UL may involve intentional disposition for the affected arm use and overcome the learned nonuse phenomenon. ${ }^{2}$ Patients in the distributed CIT were forced to use the affected UL to practice daily activities and resolve the possible difficulties that they might encounter while performing daily tasks. Accordingly, in comparison with control intervention, they may have had greater improvements in functional ability and use of the affected UL in daily life, leading to enhanced overall quality of life. In contrast, BAT did not show equivalent improvements in functional performance (AOU and QOM scores) or quality of life. The nonsignificant finding on AOU and QOM is consistent with the study by Richards et al. ${ }^{18}$ It is possible that the BAT group may have more use of the unaffected UL during daily activities because this therapy did not emphasize forced use of the affected arm. Furthermore, bilaterally symmetrical tasks of daily living are actually fewer in number than bilaterally complementary tasks, in which the 2 arms have different functions and movement patterns. ${ }^{26}$ Patients in the BAT group may have experienced fewer functional tasks relevant for real-world life during the training and may not be able to incorporate gains in motor function into daily use.

There is no significant difference in the overall FIM score between the 3 groups, similar to the findings of the previous studies ${ }^{4,10}$ where no significant differences were found between the CIT and control groups. It might be that some aspects such as sphincter control, cognition, communication of the FIM, representing at least $50 \%$ of the total score, are possibly not expected to change as a result of a voluntary, physical rehabilitation intervention. The finding of this study is in contrast with the previous study ${ }^{9}$ showing better improvement in the FIM score after CIT than after the control intervention. Participant characteristics might account for this discrepancy. Whereas the previous study employed older patients aged $>65$ years, this study employed both younger and elderly patients.

As an interesting finding of the study, the distributed CIT, but not BAT, facilitated better locomotion reflected by the FIM than the control intervention, while none of these 3 treatment protocols focused on locomotion training. Dobkin ${ }^{44}$ suggests that a nonfunctioning arm may influence walking ability in stroke patients. The affected arm may act as a dead weight, dragging on and changing trunk alignment, thereby making it difficult to balance on the affected leg. The impaired walking ability may then result in difficulties in locomotion. Increased functional use of the affected hand after distributed CIT may have relieved the negative impact on trunk alignment and balance control, and thus resulted in improved locomotion. ${ }^{44}$ Although most aspects of the FIM did not improve after intervention, descriptive statistics showed improved locomotion after distributed CIT and reduction of the variability of selfcare after the interventions, suggesting that these improvements were not a function of natural recovery.

The distributed CIT group obtained greater gains in the overall and some individual domains of quality of life (eg, $\mathrm{ADL} / \mathrm{IADL}$ and hand function) than the BAT and control groups. This finding is largely consistent with previous studies that reported the benefits of CIT for improving the overall or physical domains of quality of life. ${ }^{5,9}$ Because the distributed CIT program focused on the repeated practice of functional tasks, patients may be more able to improve on physical performance and daily function. In contrast, the change in quality of life score was small in the BAT group, and this may be associated with the restricted effects of BAT on use of the affected UL and daily function.

This comparative trial extended prior research on stroke motor rehabilitation by studying BAT, distributed CIT, and control intervention with intensive treatment schedule for 2 hours/day, 5 days/week, for 3 weeks. To our knowledge, no prior studies have used similar protocols with similar treatment intensity for comparison among these rehabilitation therapies. Some previous studies ${ }^{9,11}$ compared distributed CIT with control intervention with the same treatment intensity. Other studies $^{19-22}$ examined the effects of BAT involving repetitive practice on functional tasks similar to the present study but did not contrast BAT with distributed CIT. Furthermore, these studies on BAT used single-case study designs,${ }^{20,22}$ employed less treatment intensity, ${ }^{20-22}$ or adopted both symmetrical and sequential movements as training tasks in the BAT group, ${ }^{19}$ which were different from the present study. With respect to the study design and sample size, previous studies ${ }^{3,4,7-13,17}$ used either a nonrandomized controlled trial (1 group pretest and posttest) or a randomized controlled trial with participant number ranging from 9 to 21 per group. In a randomized controlled trial, Wolf et al ${ }^{5}$ employed a large sample size (106 or 110 participants for each group). Nevertheless, Wolf et al did not report the treatment duration for each session in the control group. The present study employed 20 participants for each group, falling into the range of participant numbers in most previous studies. Duration of therapy was kept equal for the treatment groups in our study.

The overall findings of this study suggest that BAT might be a more compelling approach to improving UL motor skills 
than distributed CIT, especially in the proximal parts. However, distributed CIT could be an optimal approach if improving nonuse of the affected arm and increasing daily function and quality of life are the primary treatment goals. The finding that the distributed CIT group exhibited greater improvements in locomotion than the BAT or control intervention groups suggests the relevance of distributed CIT for improving this important aspect of function in addition to overcoming learned nonuse of the UL. Future CIT trials may include measures of mobility and functional ambulation.

A few limitations to this study warrant consideration. First, these study findings were based on a small trial with a varied sample representing a small percentage of all eligible patients. Caution should be exercised when generalizing the results beyond the study. Further research should recruit a larger sample to validate the findings of the present study. Second, the treatment effects were measured immediately after treatment. Therefore, it is unclear whether the benefits of intervention are retained after treatment. In addition, the lack of improvement after BAT in daily function or functional use of the affected arm might be reversed over time. Further study is needed to evaluate the immediate and long-term effects of treatment. Third, this study did not explore the motor control mechanism after intervention. Future research may use instrumental kinematic evaluation, a sensitive tool for evaluating upper extremity movements after stroke, ${ }^{45,46}$ to examine the effects of intervention on motor control strategy and the mechanism underlying the change.

Although the present study has demonstrated significant and moderate to large effects of distributed CIT and BAT on clinical measures of daily function and quality of life, further research is needed to explore the changes in the real lives of the patients after the treatments. For example, future research may use the accelerometry system ${ }^{47}$ to monitor body motion, activity, and energy expenditure of the patients in free-living conditions. An additional line of research pertains to the study of the combined effects of 2 training programs (eg, BAT coupled with distributed CIT) versus a single program (eg, BAT or distributed CIT) in different types of stroke patients. ${ }^{25,26}$ Future research may also assess individual factors that may affect outcomes after the intervention (eg, stroke severity, side of hemiplegia, level of motor impairment, and motivation for treatment participation). Such research may reveal prognostic factors for each intervention type as it relates to outcome prediction and patient selection.

\section{Conclusion}

This study compared distributed CIT to BAT based on comprehensive outcome measures. Although both protocols improved overall UL motor skills, BAT was superior to distributed CIT in improving motor function of the proximal UL. However, the beneficial effects of BAT may not translate into improved functions of daily activities and quality of life. In contrast, distributed CIT demonstrated greater gains in functional use of the affected UL in daily life and improved functional independence (eg, locomotion) and quality of life (eg, ADL/IADL domains) relative to BAT and control intervention. These findings emphasize the need to take domains of outcome measures into consideration when comparing stroke rehabilitation programs.

\section{Acknowledgments}

This project was supported in part by the National Science Council (NSC-95-2314-B-002-225-MY2, NSC-96-2320-B182-029, NSC-96-2628-B-002-033-MY2, and NSC-97-2314B-002-008-MY3) and the National Health Research Institutes (NHRI-EX97-9742PI) in Taiwan.

\section{References}

1. Cauraugh JH, Summers JJ. Neural plasticity and bilateral movements: a rehabilitation approach for chronic stroke. Prog Neurobiol. 2005;5: 309320 .

2. Hakkennes S, Keating JL. Constraint-induced movement therapy following stroke: a systematic review of randomised controlled trials. Aust J Physiother. 2005;51:221-231.

3. Bonifer NM, Anderson KM, Arciniegas DB. Constraint-induced movement therapy after stroke: efficacy for patients with minimal upper-extremity motor ability. Arch Phys Med Rehabil. 2005;86:1867-1873.

4. Sawaki L, Butler AJ, Leng X, et al. Constraint-induced movement therapy results in increased motor map area in subjects 3 to 9 months after stroke. Neurorehabil Neural Repair. 2008;22:505-513.

5. Wolf SL, Winstein CJ, Miller JP, et al. Effect of constraint-induced movement therapy on upper extremity function 3 to 9 months after stroke. JAMA. 2006;296:2095-2104.

6. Page SJ, Levine P, Sisto S, Bond Q, Johnston MV. Stroke patients' and therapists' opinions of constraint-induced movement therapy. Clin Rehabil. 2002;16:55-60.

7. Dettmers C, Teske U, Hamzei F, Uswatte G, Taub E, Weiller C. Distributed form of constraint-induced movement therapy improves functional outcome and quality of life after stroke. Arch Phys Med Rehabil. 2005;86: 204-209.

8. Page SJ, Levine P, Leonard A, Szaflarski JP, Kissela BM. Modified constraint-induced therapy in chronic stroke: results of a single-blinded randomized controlled trial. Phys Ther. 2008;88:333-340.

9. Wu CY, Chen CL, Tsai WC, Lin KC, Chou SH. A randomized controlled trial of modified constraint-induced movement therapy for elderly stroke survivors: changes in motor impairment, daily functioning, and quality of life. Arch Phys Med Rehabil. 2007;88:273-278.

10. Boake C, Noser E, Ro T, et al. Constraint-induced movement therapy during early stroke rehabilitation. Neurorehabil Neural Repair. 2007;21: 14-24.

11. Wu CY, Lin KC, Chen CL, Chen IH, Hong WH. Effects of modified constraint-induced movement therapy on movement kinematics and daily function in patients with stroke: a kinematic study of motor control mechanisms. Neurorehabil Neural Repair. 2007;21:460-466.

12. Schaechter JD, Kraft E, Hilliard TS, et al. Motor recovery and cortical reorganization after constraint-induced movement therapy in stroke patients: a preliminary study. Neurorehabil Neural Repair. 2002;16: 326338.

13. Whitall J, McCombe Waller S, Silver KH, Macko RF. Repetitive bilateral arm training with rhythmic auditory cueing improves motor function in chronic hemiparetic stroke. Stroke. 2000;31:2390-2395. 
14. Summers JJ, Kagerer FA, Garry MI, Hiraga CY, Loftus A, Cauraugh JH. Bilateral and unilateral movement training on upper limb function in chronic stroke patients: a TMS study. J Neurol Sci. 2007;252:76-82.

15. Stewart KC, Cauraugh JH, Summers JJ. Bilateral movement training and stroke rehabilitation: a systematic review and meta-analysis. J Neurol Sci. 2006;244:89-95.

16. Hesse S, Werner C, Pohl M, Rueckriem S, Mehrholz J, Lingnau ML. Computerized arm training improves the motor control of the severely affected arm after stroke: a single-blinded randomized trial in two centers. Stroke. 2005;36:1960-1966.

17. Luft AR, McCombe Waller S, Whitall J, et al. Repetitive bilateral arm training and motor cortex activation in chronic stroke: a randomized controlled trial. JAMA. 2004;292:1853-1861.

18. Richards LG, Senesac CR, Davis SB, Woodbury ML, Nadeau SE. Bilateral arm training with rhythmic auditory cueing in chronic stroke: not always efficacious. Neurorehabil Neural Repair. 2008;22:180-184.

19. Desrosiers J, Bourbonnais D, Corriveau H, Gosselin S, Bravo G. Effectiveness of unilateral and symmetrical bilateral task training for arm during the subacute phase after stroke: a randomized controlled trial. Clin Rehabil. 2005;19:581-593.

20. Mudie MH, Matyas TA. Can simultaneous bilateral movement involve the undamaged hemisphere in reconstruction of neural network damaged by stroke? Disabil Rehabil. 2000;22:23-37.

21. Mudie MH, Matyas TA. Responses of the densely hemiplegic upper extremity to bilateral training. Neurorehabil Neural Repair. 2001;15: 129-140.

22. Lewis GN, Byblow WD. Neurophysiological and behavioral adaptations to a bilateral training intervention in individuals following stroke. Clin Rehabil. 2004;18:48-59.

23. Lum PS, Burgar CG, Shor PC, Majumdar M, Van der Loos M. Robotassisted movement training compared with conventional therapy techniques for the rehabilitation of upper-limb motor function after stroke. Arch Phys Med Rehabil. 2002;83:952-959.

24. Volpe BT, Lynch D, Rykman-Berland A, et al. Intensive sensorimotor arm training mediated by therapist or robot improves hemiparesis in patients with chronic stroke. Neurorehabil Neural Repair. 2008;22:305-310.

25. Whitall J. Stroke rehabilitation research: time to answer more specific questions? Neurorehabil Neural Repair. 2004;18:3-8.

26. McCombe Waller S, Whitall J. Bilateral arm training: why and who benefits? NeuroRehabilitation. 2008;23:29-41.

27. Wolf SL. Revisiting constraint-induced movement therapy: are we too smitten with the mitten? Is all nonuse "learned"? and other quandaries. Phys Ther. 2007;87:1212-1223.

28. Mudie MH, Matyas TA. Upper extremity retraining following stroke: effects of bilateral practice. J Neurol Rehabil. 1996;10:167-184.

29. Brunnstrom S. Movement Therapy in Hemiplegia. New York: Harper \& Row; 1970.

30. Taub E. Constraint-induced movement therapy and massed practice [letter to the editor]. Stroke. 2000;31:983.
31. Teng EL, Chui HC. The modified Mini-Mental State Exam. J Clin Psychiatry. 1987;48:314-318.

32. Portney LG, Watkins MP. Foundations of Clinical Research: Application to Practice. 3rd ed. Upper Saddle River, NJ: Prentice Hall Health; 2009.

33. Duncan PW, Propst M, Nelson SG. Reliability of the Fugl-Meyer assessment of sensorimotor recovery following cerebrovascular accident. Phys Ther. 1983;63:1606-1610.

34. Gladstone D, Danells C, Black SE. The Fugl-Meyer Assessment of motor recovery after stroke: a critical review of its measurement properties. Neurorehabil Neural Repair. 2002;16:232-240.

35. Kidd D, Stewart G, Baldry J, et al. The Functional Independence Measure: a comparative validity and reliability study. Disabil Rehabil. 1995;17: 10-14.

36. Law M. Self-care. In: Van Deusen J, Brunt, D, eds. Assessment in Occupational and Physical Therapy. Philadelphia, PA: W. B. Saunders; 1997:421-433.

37. Sterr A, Elbert T, Berthold I, Kölbel S, Rockstroh B, Taub E. Longer versus shorter daily constraint-induced movement therapy of chronic hemiparesis: an exploratory study. Arch Phys Med Rehabil. 2002;83:1374-1377.

38. Uswatte G, Taub E, Morris D, Light K, Thompson PA. The Motor Activity Log-28: assessing daily use of the hemiparetic arm after stroke. Neurology. 2006;67:1189-1194.

39. Duncan PW, Bode RK, Min Lai S, Perera S; Glycine Antagonist in Neuroprotection Americans Investigators. Rasch analysis of a new strokespecific outcome scale: the Stroke Impact Scale. Arch Phys Med Rehabil. 2003;84:950-963.

40. Cohen J. Statistical Power Analysis for the Behavioral Sciences. 2nd ed. Hillsdale, NJ: Lawrence Erlbaum; 1988.

41. Sabes P. The planning and control of reaching movements. Curr Opin Neurobiol. 2000;10:740-746.

42. McCombe Waller S, Forrester LW, Villagra F, Whitall J. Intracortical inhibition and facilitation with unilateral dominant, unilateral nondominant and bilateral movement tasks in left and right handed adults. $J$ Neurol Sci. 2008;269:96-104.

43. Stinear JW, Byblow WD. Disinhibition in the human motor cortex is enhanced by synchronous upper limb movements. J Physiol. 2002;543: 307-316.

44. Dobkin BH. Confounders in rehabilitation trials of task-oriented training: lessons from the designs of the EXCITE and SCILT multicenter trials. Neurorehabil Neural Repair. 2007;21:3-13.

45. Caimmi M, Carda S, Giovanzana C, et al. Using kinematic analysis to evaluate constraint-induced movement therapy in chronic stroke patients. Neurorehabil Neural Repair. 2008;22:31-39.

46. Wagner JM, Rhodes JA, Patten C. Reproducibility and minimal detectable change of three-dimensional kinematic analysis of reaching tasks in people with hemiparesis after stroke. Phys Ther. 2008;88:652-663.

47. Uswatte G, Giuliani C, Winstein C, Zeringue A, Hobbs L, Wolf SL. Validity of accelerometry for monitoring real-world arm activity in patients with subacute stroke: evidence from the extremity constraint-induced therapy evaluation trial. Arch Phys Med Rehabil. 2006;87:1340-1345.

For reprints and permission queries, please visit SAGE's Web site at http://www.sagepub.com/journalsPermissions.nav 